\title{
Evaluation of Kidney Dose in Neuroendocrine Tumors Patients after Peptide Receptor Radionuclide Therapy using ${ }^{177}$ Lu-DOTATATE
}

\author{
N.R. Hidayati ${ }^{{ }^{*}}$, A. Poon ${ }^{2}$, K. Willowson ${ }^{3}$, E. Eslick ${ }^{4}$, H. Ryu ${ }^{4}$ and D.L. Bailey ${ }^{3,4}$ \\ ${ }^{I}$ Center for Technology of Radiation Safety and Metrology, National Nuclear Energy Agency, \\ Jl. Lebak Bulus Raya No. 49, Jakarta 12440, Indonesia \\ ${ }^{2}$ Department of NuclearMedicine, Austin Health, Melbourne 3084, Australia \\ ${ }^{3}$ Department of Nuclear Medicine, Royal North Shore Hospital, NSW 2065, Australia \\ ${ }^{4}$ University of Sydney, NSW 2006, Australia
}

\section{ARTICLE INFO}

Article history:

Received 30 November 2018

Received in revised form 15 July 2019

Accepted 15 July 2019

Keywords:

Neuroendocrine tumors

Kidney radiation dose

${ }^{177}$ Lu-DOTATATE

\begin{abstract}
A B S T R A C T
Radiation dose to the kidneys (kidney dose) in ${ }^{177}$ Lu-DOTATATE - Peptide Receptor Radionuclide Therapy (PRRT) is considered to be the main potential sideeffect from the treatment. Prospective assessment of kidney radiation dose can be made with SPECT, however, this requires an intensive imaging regime over a number of days. For this reason, a retrospective investigation of kidney uptake using quantitative SPECT was performed. The aim of the study was to compare the estimated radiation dose to kidneys for each cycle. Seventeen patients treated with ${ }^{177} \mathrm{Lu}$-DOTATATE for metastatic neuro-endocrine tumors had full imaging for each of their treatment cycles on a Siemens Intevo SPECT/CT gamma camera. One course of treatment consisted of 3 or 4 cycles approximately 8 weeks apart spanning 6 months. SPECT/CT scans of the abdomen were acquired at 3 time points (4, 24 and 96-120 hours) after administration of $\sim 7.8 \mathrm{GBq}$ of ${ }^{177}$ Lu-DOTATATE. Nine patients received three cycles in total and eight patients had four cycles. Volumes of interest (VOIs) were defined on a CT scan co-registered with the SPECT images and repeated over all time points, to give the radioactivity in the kidneys. Whole organ dosimetry was estimated using OLINDA/EXM using an exponential clearance model. This gives an estimate of radiation absorbed dose to kidneys, in the unit of absorbed dose of organ per administered activity $(\mathrm{Gy} / \mathrm{GBq})$ for each treatment cycle. The mean of the 3 or 4 cycles and variation can then be determined. The result shows that the average kidney radiation dose was $0.23 \mathrm{~Gy} / \mathrm{GBq}$ (range: $0.06-0.42$ ) and the average variation between cycles for all subjects expressed as a percentage was $(12.5 \pm 7.8) \%$ (median: $11.4 \%$, range: $1.8 \%-29.4 \%$ ). From this study, it can be concluded that the estimated radiation dose to the kidneys for PRRT shows good reproducibility (typically $<20 \%$ variation) within an individual across all cycles within one course of treatment (up to 4 cycles). The errors introduced by assuming that the dosimetry estimate per unit $\mathrm{GBq}$ administered from the initial cycle could be used for subsequent cycles within a course are unlikely to contribute significantly to the overall estimate of radiation burden and are considered to be safe.
\end{abstract}

(C) 2019 Atom Indonesia. All rights reserved

\section{INTRODUCTION}

Neuroendocrine tumors (NETs) are heterogeneous neoplasms which are hormonally active with variable natural history and prognosis. These tumors are characterized by their endocrine metabolism, histological pattern, and the presence of

\footnotetext{
*Corresponding author.

E-mail address: inn98@batan.go.id

DOI: https://doi.org/10.17146/aij.2019.910
}

secretory granules as well as the ability to produce biogenic amines and polypeptide hormones and originating in the small intestine or in the pancreas, which are often metastatic at the time of initial diagnosis [1-4]. In Indonesia, the data of NET patients is lacking in number. For example in RSUD Soetomo Hospital - Surabaya, there are only five cases have been found during 2008-2010 [5]. However, in the US, the number of NETs incidence has been reported increasing about 1.09 per 100,000 
persons in 1973 and increased to 6.98 per 100,000 persons by 2012 [6]. NET incidence also has been reported as a global burden for worldwide cancer incidence [7] which has increased over the past two decades, with the lungs, pancreas, small intestine, rectum, small bowel and colon as the primary sites. Figure 1 presents the statistic of NET incidence for last five decades in the US. From the figure, the number of NETs incidence has impeded the incidence of all malignant neoplasms in the US in the end of 2012.

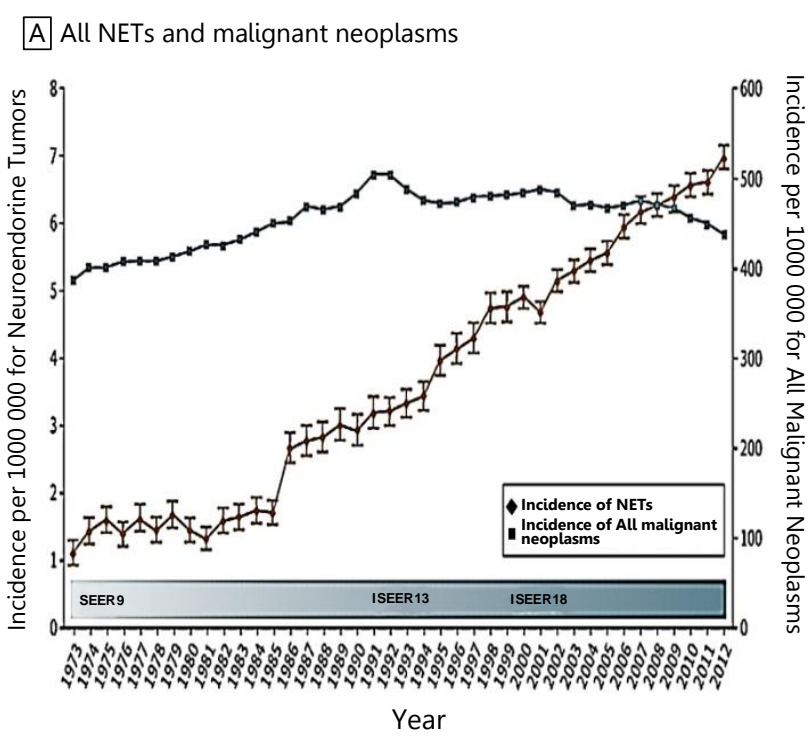

Fig. 1. The statistic of NET incidences in the US from 1973 to 2102 [6]

In 1993, the Erasmus MC hospital in Rotterdam has successfully treated the NET patients with Peptide Receptor Radionuclide Therapy (PRRT) for the first time [8]. Since then, PRRT have been used for treating NET patients. Despite the benefit of PRRT, there were preclinical studies which have shown that kidneys are the main potential side-effect from PRRT [9,10]. Moreover, some clinical studies have reported the similar finding of the effect of PRRT on kidneys [11-15]

To evaluate the effect of PRRT on kidneys, prospective assessment of kidneys can be made with gamma camera and hybrid imaging system such as Single Photon Emission Computed Tomography (SPECT or SPECT/CT) method [16]. However this requires an intensive imaging regime over a number of days.

For this reason, a retrospective investigation of kidney uptake using quantitative SPECT was performed for evaluating the kidney radiation dose on NET patients who have been treated with ${ }^{177}$ Lu-DOTATATE for 3-4 cycles. The aim of the study was to compare the estimated radiation dose to kidneys for each cycles and evaluating the dose during the treatment, so that the kidney dose for next cycles can be predicted and reduce the scan timing. Since this is a retrospective study, the ethical proposal is not performed. However, all patients have been given Informed Consent to the ${ }^{177} \mathrm{Lu}$ procedure and subsequent use of their imaging and medical data for research, teaching and training purposes, by following Australian Law regarding the informed consent [17]

\section{Peptide receptor radionuclide therapy (PRRT)}

Peptide receptor radionuclide therapy (PRRT) is a targeted radionuclide therapy which specifically uses radiolabeled peptides as biological targeting agent designed to deliver cytotoxic levels of radiation dose to cancer cells. Some radionuclides have been identified as useful for the therapy, such as : ${ }^{111} \mathrm{In},{ }^{90} \mathrm{Y},{ }^{177} \mathrm{Lu},{ }^{67} \mathrm{Cu},{ }^{47} \mathrm{Sc},{ }^{161} \mathrm{Yb},{ }^{225} \mathrm{Ac}$, and ${ }^{213} \mathrm{Bi}[18]$

${ }^{177} \mathrm{Lu}$ emits $\beta$ electrons with linear energy transfer values of about $0.2 \mathrm{keV} / \mu \mathrm{m}$, and has a range in tissue of several millimeters. ${ }^{177} \mathrm{Lu}$ decays with a half-life of $6.71 \mathrm{~d}$ by emission of $\beta$ - particles with $\mathrm{E}_{\max }$ of $497 \mathrm{keV}(78.6 \%), 384 \mathrm{keV}(9.1 \%)$ and $176 \mathrm{keV}(12.2 \%)$ to stable ${ }^{177} \mathrm{Hf}$. For this reason, ${ }^{177} \mathrm{Lu}$ is the most widely used radioisotope for in-vivo procedures and targeted radionuclide therapy, because of its favorable decay characteristics [19].

The characteristic of ${ }^{177} \mathrm{Lu}$ penetration has been considered as an ideal tool for delivering energy to small volumes and lesion. From the production and labelling aspects, ${ }^{177} \mathrm{Lu}$ has a good possibility to be labelled with peptide and protein radiolabeling of antibodies that have slow targeting kinetics. In term of physical half-life, ${ }^{177} \mathrm{Lu}$ is desirable because it provides more time for logistical arrangement from the reactor production to the imaging/therapy facilities[20]. In addition, it has been reported that ${ }^{177} \mathrm{Lu}$-DOTATATE has better residence time than ${ }^{177}$ Lu-DOTATATOC [DOTA0,Tyr3] octreotide. This is because ${ }^{177} \mathrm{Lu}$ - DOTATATE has higher ratio of absorbed dose between target (tumors) and non targets, especially for dose limiting organ [21].

\section{Kidney dose}

Dosimetry assessment for radionuclide therapy has been a raising concern, especially in PRRT in which the kidneys have been identified as the primary critical organ. This concern is caused by the fact that small peptides are filtered through the 
glomerular capillaries and are reabsorbed by the proximal tubular cells [22]. Since the limiting dose for PRRT has not been standardized, most protocols in radiotherapy have adopted the limiting dose for kidneys from external beam radiation therapy at about 30 Gy (or less) [23]. The limiting dose was addressed for protecting the kidneys from the failure due to excessive radiation, called as radiation nephrotoxicity. For this reason, an evaluation of uptake in kidneys need to be performed to optimize the radiation protection to patients in PRRT $[23,24]$.

\section{EXPERIMENTAL METHODS}

The work in this study was carried out in the Department of Nuclear Medicine, Royal North Hospital Sydney, Australia. The methodology for performing the work was following the method which has been described in previous study by Bailey D.L, et al [25]

The investigation was performed for consecutive studies of 17 patients with metastatic neuro-endocrine tumors (8 males, 9 females) who underwent PRRT with ${ }^{177}$ Lu-DOTATATE. The PRRT was performed by standard protocol with administration of a mean activity of $7.76 \pm 0.36 \mathrm{GBq}$ $(209 \pm 9.1 \mathrm{mCi}){ }^{177} \mathrm{Lu}$-octreotate per treatment cycle. One course of treatment consisted of 3 or 4 cycles approximately 8 weeks apart that spanning 6 months.

The ${ }^{177} \mathrm{Lu}$ that has been used in this work was produced in a nuclear reactor by an $(\mathrm{n}, \gamma)$ reaction (ITG, Isotope Technologies Garching $\mathrm{GmbH}$, Germany and the Australian Nuclear Science and Technology Organization (ANSTO), Australia). The ${ }^{177} \mathrm{Lu}$-octreotate is referred to DOTATATE (DOTA0-(Tyr3)-octreotate where DOTA $=1,4,7,10$ tetra-azacyclododecane-1,4,7,10- tetraacetic acid) (Auspep, Melbourne, Australia). During the administration of ${ }^{177}$ Lu-DOTATATE, patients were accompanied by a 3-h amino acid infusion in order to provide protection for the kidneys from radiation.

The image acquisition was performed using Siemens Intevo SPECT/CT gamma camera at 3 time points (4, 24 and 96-120 hours) after administration. The images were analyzed using in-house developed software using a high-level scientific programming language (IDL, Exelis Visual Information Solutions, Herndon, VA, USA) on a dedicated nuclear medicine workstation (HERMES, Nuclear Diagnostics, Stockholm, Sweden). The volumes of interest (VOI) were defined on a CT scan coregistered with the SPECT images and repeated over all time points, to acquire the uptake of ${ }^{177}$ Lu-DOTATATE in the kidneys.
To perform whole organ dosimetry, OLINDA/EXM ver.1 was used by performing biexponential clearance model. OLINDA/EXM is the software which has been approved by US FDA as a tool for internal dosimetry assessment in nuclear medicine [26]. The result of OLINDA/EXM is in the unit of absorbed dose of organ per administered activity $(\mathrm{mSv} / \mathrm{MBq})$ or $\mathrm{rem} / \mathrm{mCi}$. Hence in this study, the absorbed dose of kidneys for each treatment is given for each treatment cycle. The mean of the 3 or 4 cycles and variation can then be determined.

\section{RESULTS AND DISCUSSION}

In this study, the evaluation process has been started with acquiring serial scans for 4, 24 and 96120 hours after the administration of ${ }^{177} \mathrm{Lu}-$ DOTATATE. In each time points, the kidneys have been delineated as VOI using the HERMES Workstation, and registering the VOI with CT images. In this step, the VOI of kidneys is presenting as functional (physiological) data, which needs to be registered into CT images. The HERMES work station makes it possible to combine both functional and anatomical data into quantitative value which are useful for investigating the organ dose in PRRT.

Figure 2 showing the screen shoot of images from HERMES workstation, in which the VOI of kidneys and its registration has been performed as 3D quantification imaging. The result of quantification in this step is the percentage of uptake of ${ }^{177}$ Lu-DOTATATE in kidneys to be used as the input into OLINDA/EXM.

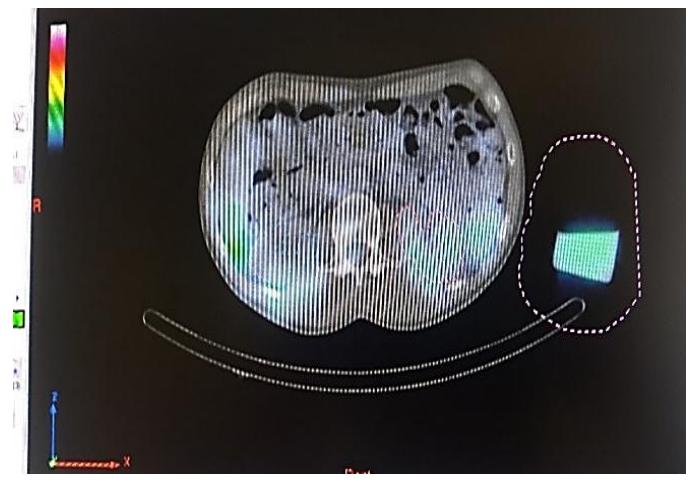

Fig. 2. The screen shoot of HERMES workstation for ${ }^{177}$ Lu-DOTATATE patients.

To utilize OLINDA/EXM as a tool for internal dosimetry calculation, it is important that the data consist of at least 3 time points. For this reason, there were some patients data which are excluded from the study, since it has only 2 time points. By using the fitting menu and using the biexponential function, the residence time of ${ }^{177} \mathrm{Lu}$ - 
DOTATATE in the kidneys and the kidney dose for seventeen patients who underwent 3-4 treatment cycles has been obtained. Figure 3 showing the sample screen of OLINDA/EXM in fitting menu.

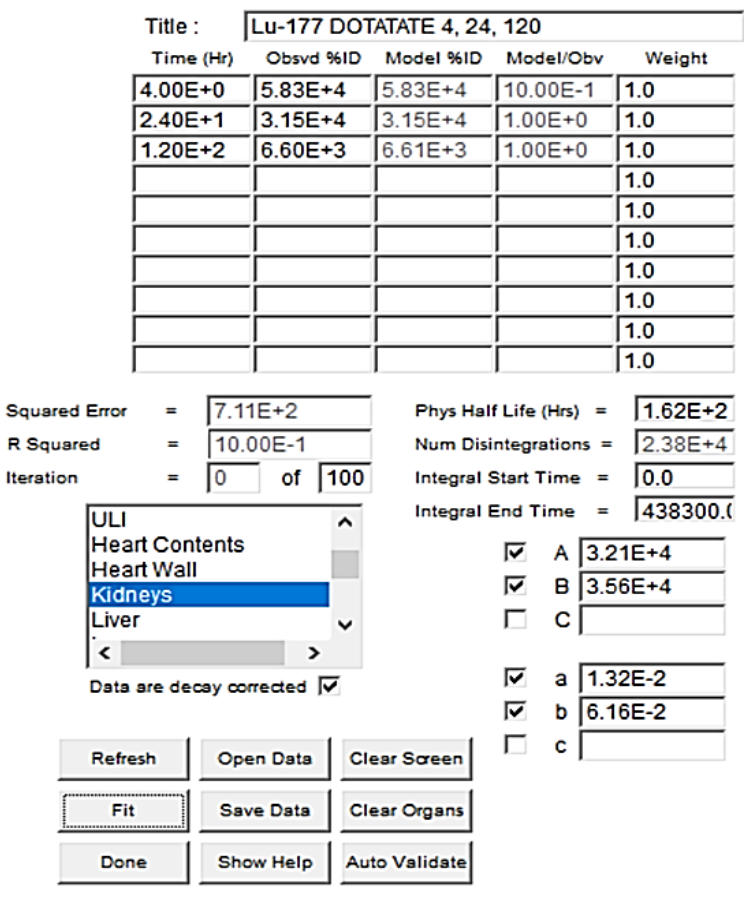

Fig. 3. Sample screen from OLINDA/EXM code, fitting data for ${ }^{177} \mathrm{Lu}$.

Table 1. Variation of kidney dose of NET patients with ${ }^{177} \mathrm{Lu}$-DOTATATE between cycles.

\begin{tabular}{ccccc}
\hline \multirow{2}{*}{ Subject } & SEX & \multicolumn{3}{c}{ CoV \% ID Kidneys } \\
\cline { 3 - 5 } & $(\mathrm{M} / \mathrm{F})$ & $(4 \mathrm{~h})$ & $(24 \mathrm{~h})$ & $(120 \mathrm{~h})$ \\
\hline $\mathrm{A}$ & $\mathrm{F}$ & $29 \%$ & $38 \%$ & $47 \%$ \\
$\mathrm{~B} 1$ & $\mathrm{M}$ & $24 \%$ & $21 \%$ & $22 \%$ \\
$\mathrm{~B} 2$ & $\mathrm{M}$ & $8 \%$ & $7 \%$ & $15 \%$ \\
$\mathrm{C} 1$ & $\mathrm{~F}$ & $19 \%$ & $27 \%$ & $30 \%$ \\
$\mathrm{C} 2$ & $\mathrm{~F}$ & $16 \%$ & $23 \%$ & $8 \%$ \\
$\mathrm{D}$ & $\mathrm{F}$ & $8 \%$ & $10 \%$ & $12 \%$ \\
$\mathrm{E}$ & $\mathrm{M}$ & $17 \%$ & $18 \%$ & $9 \%$ \\
$\mathrm{~F}$ & $\mathrm{M}$ & $8 \%$ & $4 \%$ & $13 \%$ \\
$\mathrm{G}$ & $\mathrm{F}$ & $6 \%$ & $5 \%$ & $8 \%$ \\
$\mathrm{H}$ & $\mathrm{F}$ & $18 \%$ & $16 \%$ & $35 \%$ \\
$\mathrm{I}$ & $\mathrm{M}$ & $17 \%$ & $17 \%$ & $40 \%$ \\
$\mathrm{~J}$ & $\mathrm{M}$ & $6 \%$ & $13 \%$ & $26 \%$ \\
$\mathrm{~K}$ & $\mathrm{M}$ & $7 \%$ & $6 \%$ & $4 \%$ \\
$\mathrm{~L}$ & $\mathrm{~F}$ & $8 \%$ & $10 \%$ & $2 \%$ \\
$\mathrm{M}$ & $\mathrm{F}$ & $14 \%$ & $3 \%$ & $2 \%$ \\
$\mathrm{~N}$ & $\mathrm{~F}$ & $17 \%$ & $6 \%$ & $1 \%$ \\
$\mathrm{O}$ & $\mathrm{F}$ & $7 \%$ & $6 \%$ & $6 \%$ \\
$\mathrm{P}$ & $\mathrm{M}$ & $5 \%$ & $2 \%$ & $17 \%$ \\
$\mathrm{Q}$ & $\mathrm{F}$ & $16 \%$ & $30 \%$ & $14 \%$ \\
$\mathrm{R}$ & $\mathrm{M}$ & $24 \%$ & $43 \%$ & $5 \%$ \\
\hline AVG & & $14 \%$ & $15 \%$ & $16 \%$ \\
\hline & & & &
\end{tabular}

The result of kidney dose from OLINDA/EXM was then investigated between cycles and calculated statistically. From Table 1, it can be seen that in this study the variation of kidney dose for seventeen patients who undergo the therapy, has the average kidney dose about 0.23 $\mathrm{Gy} / \mathrm{Bq}$, and the average variation between cycles for all subjects expressed as a percentage was $(12.5 \pm 7.8) \%$ (median: $11.4 \%$, range: $1.8 \%-29.4 \%$ ). This means, compared to the number of limiting dose for kidneys in external beam radiation therapy [27], the kidney dose for the patients who undergo 3-4 cycles of therapy, are in good reproducibility, safe and the dose for next cycles can be predicted.

At the moment, there is no standardized model for individualized patient dosimetry, while the evidence is increasing. Performing an individualized patient dosimetry (personalized dosimetry) has been considered to improve the efficacy of therapy and spare the health tissue [28]. Recently, the European Association of Nuclear Medicine (EANM), has published internal dosimetry task report and stated that dosimetrybased treatment planning is a mandatory to improve the effectiveness of the radionuclide therapy, by predicting the response and toxicity [29]. Beside useful as a treatment planning, there is evidence that dosimetry also can be useful for evaluation of therapy [30]. Likewise, the evaluation of kidney dose in this study is a sample how the dosimetry study in PRRT can be performed after the therapy.

Since the kidneys are the critical organs as the target of evaluation, for further similar studies, the information of kidney data would be better if the glomerular filtration rate (GFR) value of the kidneys can be contributed to support the data analysis for the patients, as it has been done in a similar study in which the grading of kidneys has been divided as chronic and acute, with GFR as supporting information [31]. This additional data might be helpful if there are any discrepancies of the data for preparing the investigation of kidneys for next cycles.

The utilization of ${ }^{177}$ Lu-DOTATATE in PRRT for NET patients has been performed worldwide, and the trend seems increasing, while in Indonesia, the data of NET patients who undergo PRRT is hardly to be found [5]. Most of the radionuclide therapy in Indonesia was performed with radioiodine [32]. This might be related to the limited availability of ${ }^{177} \mathrm{Lu}$-DOTATATE for PRRT in Indonesia and locally not provided.

Moreover, dosimetry study for radionuclide therapy patients in Indonesia has not been performed 
yet, while in some countries, dosimetry study has been suggested to be conducted as a tool for implementing radiation protection of patients who undergo radionuclide therapy [30,33]. Hence, this study is important for showing that dosimetry study in PRRT has been performed worldwide because it can improve the efficacy of therapy and protecting critical organs. Moreover, by showing the good reproducibility of kidney doses after the therapy, it will present that the radiation burden in PRRT are considered to be safe. This study may, therefore, become a starting point to the urgency of the initialization of dosimetry study for NET patients using PRRT as well as other radionuclide therapies in Indonesia.

\section{CONCLUSION}

Estimated radiation dose to the kidneys for the patients undergo PRRT with ${ }^{177}$ Lu-DOTATATE shows good reproducibility (typically $<20 \%$ variation) within an individual across all cycles within one course of treatment (up to 4 cycles). The errors introduced by assuming that the dosimetry estimate per unit GBq administered from the initial cycle could be used for subsequent cycles within a course are unlikely to contribute significantly to the overall estimate of radiation burden and are considered to be safe.

\section{ACKNOWLEDGMENT}

The author gratefully acknowledges the Head of Nuclear Medicine Department of Royal North Shore Hospital Sydney and the staff for the hospitality and assistance during the visits. The acknowledgement also has been gratefully addressed to The Non-Degree Program for Research Innovation in Science and Technology Project (RISET-PRO) from the Ministry of Research, Technology and the Higher Education (Kemenristek Dikti) for the financial support for the activities related to the development of internal dosimetry assessment in nuclear medicine in Indonesia from 2013-2015 and 2017.

\section{REFERENCES}

1. P.L. Kunz, J. Clin. Oncol. 33 (2015) 1855.

2. G.A. Watson, Y. Ahmed, S. Picardo et al., Am. J. Case Rep. 19 (2018) 710.

3. K. Öberg and A. Sundin, Front. Horm. Res. 45 (2016) 142.
4. C.J Auernhammer, C. Spitzweg, M.K Angele et al., The Lancet Diabetes and Endocrinology 6 (2018) 404.

5. M. Mahayasa and P.S. Wibowo, Jurnal Bedah Nasional 2 (2018) 28. (in Indonesian)

6. A. Dasari, C. Shen and D. Halperin et al., JAMA Oncol. 3 (2017) 1335.

7. A. Chauhan, Q. Yu, N. Ray et al., Oncotarget 9 (2018) 19245.

8. S.U. Dalm, J. Nonnekens, G.N Doeswijk et al., J. Nucl. Med. 57 (2016) 260.

9. E Schüler, N. Rudqvist and T.Z. Parris et al., Nucl. Med. Biol. 41 (2014) 238.

10. H. Bergsma, M.W Konijnenberg, W.A. Van der Zwan et al., Eur. J. Nucl. Med. Mol. Imaging 43 (2016) 1802.

11. H. Bergsma, M.W. Konijnenberg, B.L.R. Kam et al., Eur. J. Nucl. Med. Mol. Imaging 43 (2016) 453.

12. L. Bodei, M. Kidd, G. Paganelli et al., Eur. J. Nucl. Med. Mol. Imaging 42 (2015) 5.

13. A. Sabet, K. Ezzidin, U.F. Pape et al., Eur. J. Nucl. Med. Mol. Imaging 41 (2014) 505.

14. A Löser, S.M Schwarzenböck, M. Heuschkel et al., Nucl. Med. Commun. 39 (2018) 236.

15. L. Bodei, D.J. Kwekkeboom, M. Kidd et al., Seminars in Nuclear Medicine 46 (2016) 225.

16. E. Ilan, M. Sandström, C. Wassberg et al., J. Nucl. Med. 56 (2015) 177.

17. Anonymous, Informed Consent to Medical Treatment, Australian Law Reform Commission, https://www.alrc.gov.au/publications/10-reviewstate-and-territory-legislation/informed-consentmedical-treatment. Retrived in May (2014)

18. A. Dash, M.R.A. Pillai and F.F. Knapp, Nucl. Med. Mol. Imaging 49 (2015) 85.

19. A. Dash, S. Chakraborty, M.R.A Pillai et al., Cancer Biother. Radiopharm. 30 (2015) 47.

20. F. Guerriero, M.E. Ferrari, F. Botta et al., Biomed Res. Int. 2013 (2013) 1.

21. L. Kabasakal, M. AbuQbeitah, A. Aygün et al., Eur. J. Nucl. Med. Mol. Imaging 42 (2015) 1976.

22. S. Severi, I. Grassi, S. Nicolini et al., Onco Targets Ther. 10 (2017) 551.

23. J. Mortensen and H. Gutte H., Eur. J. Nucl. Med. Mol. Imaging 41 (2014) 81. 
24. J. Ahlstedt, T.A. Tran, S.E Strand et al., Int. J. Mol. Sci. 16 (2015) 30309.

25. D.L. Bailey, T.M. Hennessy, K.P. Willowson et al., EJNMMI Phys. 2 (2015) 1.

26. M.G. Stabin, R.B. Sparks, E. Crowe, J. Nucl. Med. 46 (2005) 1023.

27. M. Cremonesi, M.E. Ferrari, L. Bodei et al., J. Nucl. Med. Mol. Imaging 45 (2018) 2426.

28. M. Lassmann and U. Eberlein, J. Nucl. Med. 59 (2018) 1494.
29. D.M.V. Huizing, B.J. de With-van der Veen, M. Verheij et al., EJNMMI Res. 8 (2018) 1.

30. B. Erbas and M. Tuncel, Seminars in Nuclear Medicine 46 (2016) 462.

31. C. Stokke, P.M. Gabina, P. Solny et al., EJNMMI Phys. 4 (2017) 1.

32. H. Kartamiharja, Annual Scientific Meeting (ASM) ISNM-ISNMB-BATAN, September 2018.

33. J. Svensson, G. Berg, B. Wängberg et al., Eur. J. Nucl. Med. Mol. Imaging 42 (2015) 947. 